

\title{
Réflexions méthodologiques sur une traduction en espagnol de 'Gouverneurs de la Rosée' (1944) de Jacques Roumain, auteur haïtien
}

\author{
André Thibault
}

\section{- To cite this version:}

André Thibault. Réflexions méthodologiques sur une traduction en espagnol de 'Gouverneurs de la Rosée' (1944) de Jacques Roumain, auteur haïtien. Études de linguistique appliquée: revue de didactologie des langues-cultures, 2011. hal-02568703

\section{HAL Id: hal-02568703 \\ https://hal.science/hal-02568703}

Submitted on 10 May 2020

HAL is a multi-disciplinary open access archive for the deposit and dissemination of scientific research documents, whether they are published or not. The documents may come from teaching and research institutions in France or abroad, or from public or private research centers.
L'archive ouverte pluridisciplinaire HAL, est destinée au dépôt et à la diffusion de documents scientifiques de niveau recherche, publiés ou non, émanant des établissements d'enseignement et de recherche français ou étrangers, des laboratoires publics ou privés. 


\section{RÉFLEXIONS MÉTHODOLOGIQUES SUR UNE TRADUCTION EN ESPAGNOL DE GOUVERNEURS DE LA ROSÉE (1944) DE JACQUES ROUMAIN, AUTEUR HAÏTIEN}

Résumé : Cet article se penche sur quelques problèmes de traduction vers l'espagnol d'un ouvrage littéraire antillais, le célèbre roman Gouverneurs de la rosée (1944) de l'auteur haïtien Jacques Roumain, à partir d'une traduction publiée en 1971 à La Havane, et plus particulièrement sur le défi représenté par la traduction vers l'espagnol des antillanismes qui abondent dans ce roman. Une sélection de diatopismes ayant eu droit à une note de bas de page de la part du traducteur seront présentés sous forme d'articles de dictionnaire historico-comparatif, et leur traduction en espagnol sera commentée. L'on verra que les choix de traduction sont plus souvent "sourciers" que "ciblistes", bien que l'espagnol régional des Caraïbes permette souvent de réconcilier les deux approches. On montrera également que la méconnaissance du créole et du français d'Haïti est responsable d'un certain nombre de traductions erronées, situation à laquelle on ne peut remédier que par l'élaboration de nouveaux outils lexicographiques adaptés à la variation diatopique qui règne en francophonie.

\section{Jacques Roumain, Gouverneurs de la Rosée, 1944}

Nous allons nous pencher sur un célèbre roman de la littérature haïtienne, Gouverneurs de la Rosée, du grand auteur Jacques Roumain (Portau-Prince, 1907 ; Port-au-Prince, 1944), qui fut aussi actif en politique (il est le fondateur du Parti Communiste Haïtien). Ce roman raconte la lutte d'un homme pour obtenir que tous les habitants de son village, séparés par des haines ataviques, se réunissent pour travailler ensemble à la construction d'un système d'aqueduc pour acheminer l'eau d'une source lointaine, eau sans laquelle le village s'enfonce de plus en plus dans la misère. Le protagoniste tombe amoureux d'une jeune fille appartenant au groupe « ennemi » et finit assassiné, ce qui donne au roman des airs de Roméo et Juliette sous les Tropiques.

La traduction en espagnol que nous allons analyser s'intitule Gobernadores del rocío ; l'édition que nous avons entre les mains a été publiée en 1971 à La Havane (Cuba) dans la Colección Latinoamericana de la Casa 
de las Américas locale. ${ }^{1}$ Elle comporte un prologue de cinq pages du grand écrivain cubain Nicolás Guillén, mais curieusement le nom du traducteur ou de la traductrice n'apparait nulle part. ${ }^{2}$ Ce n'est pas un hasard si cette traduction a été publiée à Cuba : Jacques Roumain était communiste, et son livre peut être interprété comme un acte politique visant à dénoncer les conditions de vie des paysans haïtiens; mais le roman va au-delà d'une telle récupération politique, en ce sens qu'on peut très bien l'interpréter comme une allégorie de la condition humaine en général (v. Hoffmann 2003, 257-265 pour un aperçu de la réception qu'a connue l'ouvrage).

Il est très intéressant d'étudier cette traduction; d'une part, parce que Cuba et l'espagnol cubain font partie de la même aire géo-politique, historique et culturelle qu'Haïti ; d'autre part, parce que le traducteur a inclus un très grand nombre de notes de bas de page pour expliciter sa démarche et ses choix. Malheureusement, comme on va le voir, ses solutions ne sont pas toujours très heureuses. Il faut d'abord expliquer qu'à l'époque où cette traduction a été faite, il n'existait encore presque pas de dictionnaires de créole haïtien ou de publications sur le français de ce pays; et même s'ils avaient existé (on pense à Pompilus 1961), on peut douter que les traducteurs de l'époque y aient eu accès. Les éditions de Gouverneurs de la Rosée alors disponibles sur le marché n'étaient pas aussi bonnes que la toute dernière (Hoffmann 2003), qui fait autorité et commente le sens de nombreux haïtianismes. Quant aux particularismes lexicaux du français régional antillais, quelques-uns figuraient déjà à l'époque dans les grands dictionnaires français, mais encore une fois on peut douter que les traducteurs latino-américains aient pu disposer de nombreuses sources lexicographiques françaises. Ils devaient donc travailler à l'aveuglette, et faire face à des difficultés pratiquement insurmontables. Un traducteur qui s'attaquerait aujourd'hui à une pareille tâche aurait incomparablement plus de facilité à venir à bout des principaux problèmes, les publications sur le créole haïtien (et sur le français régional antillais) s'étant multipliées ces dernières décennies.

\footnotetext{
${ }^{1}$ Une nouvelle traduction, sous le titre de Gobernadores del rocio y otros textos : Selección, traducción, prólogo, notas, cronología y bibliografia, est parue en 2004 aux éditions Biblioteca Ayacucho (Vénézuéla) sous la responsabilité de Michaelle Ascencio ; merci à Alessandro Costantini d'avoir attiré notre attention sur l'existence de cette traduction, téléchargeable gratuitement à l'adresse suivante : www.bibliotecayacucho.gob.ve/fba/

${ }^{2}$ L'histoire des traductions en espagnol de Gobernadores del rocio est quelque peu nébuleuse ; voici ce que l'on peut en lire dans Ascencio 2004, XLXLI : « De Gobernadores del rocío conocemos una versión en español publicada originalmente por la Editorial Lautaro y reeditada en 1971 por el Centro Editor de América Latina. La Imprenta Nacional de La Habana publicó en 1961 una traducción que contiene un breve prólogo de Nicolás Guillén, reeditada en años posteriores. La Editorial Casa de las Américas publicó en 1971 una traducción con el prólogo de Guillén y una cronología al final. La traducción de Lautaro y la de Casa de las Américas son prácticamente idénticas. »
} 


\section{Analyse d'une sélection d'antillanismes ayant donné lieu à une note de bas de page dans la traduction}

Nous allons d'abord présenter notre analyse sous la forme d'articles lexicographiques classés par ordre alphabétique et structurés comme suit : le mot-vedette en grasse minuscule ; la catégorie grammaticale sous forme d'abréviation; une brève définition entre guillemets anglais; la citation entre guillemets français du passage dans $l^{\prime}$ 'original $^{3}$, suivie de la citation du passage dans la traduction, accompagnée du texte de la note de bas de page; une rubrique historico-comparative, assortie d'un commentaire sur les choix du traducteur; la mention du choix de traduction relevé dans Ascencio 2004, à des fins de comparaison; un bilan bibliographique détaillé. Le mot objet de l'article est souligné typographiquement par l'usage des grasses dans le corps des citations afin d'en faciliter le repérage visuel.

ago ! interj. "attention!”.

« Comme il allait sans prendre garde à ses pas, il buta contre une grosse roche qui affleurait et se rattrapa en quelques petits bonds assez ridicules.

- Ago ! dit-elle, éclatant de rire. » (p. 279)

«Como caminaba sin fijarse, tropezó él contra una roca grande que sobresalía y se agarró haciendo algunos saltitos bastante ridículos.

-¡Agú! -dijo ella, estallando de risa. » (p. 25)

Note de bas de page : « Si bien el original dice 'Agó', hemos elegido el vocablo 'Agú' empleado en Chile y Ecuador por ájájá. »

- Le traducteur ne pouvait pas savoir que cette interjection haïtienne signifie «attention!» et n'est pas du tout une onomatopée censée reproduire le bruit d'un éclat de rire. Cela n'avait donc aucun sens d'aller chercher une interjection andine paronymique car de toute façon le sémantisme n'est pas respecté. En outre, les Andes sont très loin des Antilles et le recours systématique à des régionalismes de tous les horizons déboucherait sur une sorte de patchwork incohérent qui réunirait de façon aléatoire des diatopismes de toute l'Hispanophonie sans respect pour la nature antillaise du texte de départ. Un simple iojo! (interj. esp. signifiant «attention !») aurait fait l'affaire, tout comme ;cuidado!

\footnotetext{
${ }^{3}$ Nous citons l'édition de Hoffmann 2003, 255-396.
} 
Ascencio $(2004,135)$ a maintenu « iAgo! » mais sans note de bas de page ; ce choix respecte l'original, mais le mot ne veut absolument rien dire pour un lecteur hispanophone.

BILAN BIBL. : « $\mathbf{1}$ I am here ! [voodoo] $\mathbf{2}$ attention ! $\mathbf{3}$ careful ! 》 Valdman 2007 s.v. ago $^{2}$.

ajoupa $\mathrm{n} . \mathrm{m}$. "cabane rudimentaire".

« Nous avons de la famille du côté de Boucan Corail, c'est de la famille éloignée, mais peut-être nous fera-t-elle la charité d'un morceau de terre, de quoi bâtir un ajoupa et planter un petit jardin. » (p. 339)

Note de bas de page (de Jacques Roumain) : « Case. »

« Tenemos familia del lado de Bucan Coral, son familiares lejanos pero puede ser que nos hagan la caridad de un pedazo de tierra, con que construir un ayupa y plantar un huertito. » (p. 137)

Note de bas de page : «Choza.»

- $1^{\text {res }}$ attestations : 1614 (aioupaue), 1640 (ajoupa), v. FEW 20, 56a, AIUPAUE (> «Empr. au tupi aiupaue» TLF). Exemple classique de « mot des isles », répandu des Antilles à l'Océan Indien.

En portugais brésilien, on trouve bien ajupá et tijupá (Aurélio) comme équivalents sémantiques et étymologiques du mot haïtien (il s'agit en fait d'un type lexical d'origine tupi); nous n'avons toutefois rien trouvé de semblable en espagnol. La solution proposée par le traducteur consiste donc à introduire une adaptation isolée artificielle du mot haïtien, dans l'optique des traductologues "sourciers", et sans véritable ancrage dans l'usage hispanophone caribéen.

Ascencio $(2004,199)$ traduit par choza, mot espagnol non diatopiquement marqué qui désigne plus ou moins le même référent que ajoupa.

BILAN BIBL. : Rézeau 1995, t. 1, p. 419 [1880] et t. 2, p. 26 [1881]; Boulan 1934, 197 ; Friederici 1947, 46b-47a ; « ajoupa < mot caraïbe = cabane de feuillage, abri temporaire pour les chasseurs » Jourdain 1956, 131 ; Arveiller 1963, 157 et 331 ; « hutte élevée sur des pieux et recouverte de branchages, de feuilles ou de jonc» TLF s.v. ajoup $(p) a$; «Emprunté au tupi, 'ajoupa' est d'usage courant aux Antilles dès le milieu du XVII ${ }^{\mathrm{e}}$ siècle, (cf. Du Tertre, 370, p. 140); de là il passe aux Mascareignes, importé par les marins, les colons et les agents de la Compagnie. » Chaudenson 1974, 599-600 ; «joupa (ind. car. 'aiupa', m.s.)» Faine 1974 s.v. ajoupa ; « cabane ; abri de feuillage au bord d'un champ, où l'on peut se réfugier en cas de pluie » Tourneux / Barbotin 1990 s.v. joupa ; DECOI II, 25 ; « ajoupa (langage français des Antilles); petit abri en branchages non clos 
ou, à la rigueur, construit avec une ou deux tôles ondulées » Barbotin 1995 s.v. joupa; «1. (Madag.) À l'époque coloniale, hutte de bois ou de feuillage. 2. (Haïti) Chaumière très modeste. 3. (Antilles fr.) Cabane, abri. » DUF 1997 s.v. ajoupa ; «abri léger, cabane» Telchid 1997 s.v. ajoupa (var. joupa); Breton 1665 [1999], 257 ; « cabane, paillotte, abri léger» Ludwig et al. 2002 s.v. joupa ; " (car.) hutte de branchage d'origine caraïbe que l'on construisait de manière temporaire en forêt» Confiant 2007 s.v. ajoupa (var. joupa) ; « cabane, abri » Barthèlemi 2007 s.v. ajoupa ; " [usually one-room] hut [with thatched roof, unplastered latticework walls, and dirt floor] 》 Valdman 2007 s.v. joupa (var. ajoupa); Thibault 2008, 230-231.

a tè loc. adv. "à terre".

« La voix du Simidor montait rauque et forte :

$-\boldsymbol{A}$ tè....

D'un seul coup les houes s'abattaient avec un choc sourd, attaquant le pelage malsain de la terre. » (p. 270)

«La voz de Simidor subía ronca y fuerte :

- A tiéa...

De un solo golpe los azadones caían con un choque sordo, atacando el malsano pelaje de la tierra. » (p. 7)

Note de bas de page : « En tierra...»

- La locution verbale apparaît dans le texte original en italiques ; il s'agit en fait d'un énoncé en créole. Pour le rendre, la traduction nous présente une tentative de reproduction graphique d'une prononciation considérée comme typique des populations noires des Antilles. L'ironie de la chose, c'est que l'énoncé créole n'a pas eu droit à une note de bas de page dans le texte original, alors que dans la traduction espagnole c'est le cas ; c'est donc le texte traduit qui fait l'objet d'un discours métalinguistique.

Ascencio $(2004,125)$ traduit par abajo, mot diatopiquement neutre.

BILAN BIBL. : « on the ground/floor, down » Valdman 2007 s.v. atè $\grave{3}^{3} 1$.

bayahonde $n$. m. "arbuste sauvage, épineux, de couleur verdâtre ou grisâtre, qui envahit les terrains laissés en friche".

«La même poussière que le vent rabat d'une haleine sèche sur le champ dévasté de petit-mil, sur la haute barrière de cactus rongés de vert-degris, sur les arbres, ces bayahondes rouillés. » (p. 267). 
«El mismo polvo que el viento, con su seco soplo, abate, sobre el devastado campo de mijo menor, sobre la alta barrera de los cactus roídos de cardenillo, sobre los árboles, esas bayahondas herrumbrosas. » (p. 1)

Note de bas de page : «Árbol parecido a la acacia. »

- Type lexical d'origine inconnue, mais dont l'existence en Haïti ainsi qu'en République Dominicaine et à Porto Rico laisse envisager une origine caraïbe, c'est-à-dire amérindienne. $1^{\text {res }}$ attestations : «Ces deux établissemens dépérissent, le premier surtout; car les Bayahondes se sont déjà emparés de la moitié du terrain mis en culture, il y a deux ans environ. », passage en français tiré de Accounts and papers; seven volumes. (7.)- relating to Hayti; Mexico ; Swan River Settlement ; also, Third report on criminal and civil justice in the West Indies, \&c. Sesion 5 February-2 June 1829, p. 105 (GRL) ${ }^{4}$; «Enfin, qu'est-ce qui paralyse notre industrie ? Qui laisse croître les bayahondes dans nos champs et fait de nos villes sans commerce, le séjour de la pauvreté ?» 18 mars 1831, lettre de Benjamin Fruneau dans la Feuille du Commerce (Haïti) citée dans Thomas Madiou, Histoire d'Haïti : 1827-1843, 1843 [reprint 1988], p. $88(\mathrm{GRL})^{5}$.

Le traducteur s'en est bien sorti : il a d'une part utilisé le mot espagnol de même origine utilisé en République Dominicaine pour désigner le même référent, bayahonda; il a en outre expliqué dans une note de bas de page qu'il s'agit d'une plante semblable à l'acacia.

Ascencio $(2004,121)$ traduit par cujies («Col. y Ven. aromo» DRAE 2001 ; aromo «árbol de la familia de las Mimosáceas, especie de acacia $[\ldots] \gg$ ibid.). Il s'agit d'une traduction plus cibliste destinée au lectorat colombien et vénézuélien.

BILAN BIBL. : ø Friederici 1947 ; ø Arveiller 1963 ; TLF ; ø DCECH ; Telchid 1997 ; ø DRAE 2001 ; ø GR 2008 ; ø NPR 2008. - « arbuste sauvage à feuilles pinnées (prosopis L.) » Pompilus 1961, 57, 143 ; « especie de acacia silvestre (Rep. Dom. = PR)» Richard (coord.) 2000 s.v. bayahonda $\mathrm{n}$. f. ; " plante haïtienne ; formation végétale sèche » Hénane 2004 (qui l'atteste chez Aimé Césaire); « [angl.] mesquite » Valdman 2007 s.v. bayawonn (bayahonn).

${ }^{4}$ Livre scanné et consultable intégralement.

${ }^{5}$ Livre scanné et consultable intégralement.

${ }^{6}$ Le mot anglais mesquite, donné comme équivalent par Valdman 2007, est ainsi défini dans le OED en ligne : "Any of various thorny, pinnate-leaved, thicket-forming trees and shrubs of the genus Prosopis (family Mimosaceae (Leguminosae)), characteristic of arid regions of Mexico and the south-western United States ». 
cadaches n. pl. "plantes piquantes, utilisées pour délimiter des enclos".

«Le vallon s'étendait au pied du morne. Les eaux dévalant des hauteurs l'avaient raviné et, par la pente, sa terre délavée avait été se perdre au loin. Les os des pierres perçaient sa peau maigre et les cadaches, qui sont parmi les plantes des araignées toutes velues de piquants, l'avaient envahi. » (p. 295)

«El valle se extendía al pie del cerro. Las aguas que descendían de las alturas lo habían arrollado y, por la pendiente, su tierra deslavada había ido a perderse a lo lejos. Los huesos de las piedras perforaban su piel escuálida y los cadaches que son entre las plantas como arañas todas vellosas de espinas, la habían invadido.» (p. 55)

Note de bas de page : «Enebro.»

- Type lexical extrêmement rare. Dans GRL, on trouve une toute petite poignée d'attestations, toutes haïtiennes, dont celle de J. Roumain. V. cidessous :

"Ces maisons étaient couvertes généralement en essentes, percées de lucarnes et de fenêtres sur un comble habitable. Des clôtures en pieux pointus ou en cadache fermaient quelquefois des terrains [...].» Rodolphe Charmant, La vie incroyable d'Alcius, Ateliers Fardin, Port-auPrince, 1981, p. 34 (l'auteur a vécu de 1844 à 1934 ; cette publication est donc posthume et l'attestation peut être datée « av. $1934 »)$.

" Des cadaches aussi, ces 'piquants' qui éclatent sous l'action de la chaleur de midi, pénétrant dans la chair pour n'en sortir qu'avec une partie. » Victor Bastien, En Haïti dans la vie rurale: scènes vécues, Comité haïtien pour la promotion et la diffusion des œuvres littéraires, Port-auPrince, 1979, p. 242.

Selon le dictionnaire de Valdman, le mot désigne aussi un acte de propriété se référant à un terrain ; il s'agit vraisemblablement du mot français cadastre. Le cadastre délimite les frontières des propriétés foncières. Or, comme la citation de R. Charmant ci-dessus nous apprend que les clôtures qui délimitent les terrains sont parfois faites de «cadache», il faut peut-être voir là l'origine métonymique du sens de "plantes piquantes ». Cette hypothèse est étayée par des attestations du type fr. cadasse / cr. kadas désignant des plantes piquantes, ce qui donne à entendre que cadasse et cadache ne sont que deux avatars phonétiques du même type lexical (pour la variation -sse et -che en créole haïtien, v. Fattier 1998, t. 1, p. 103 et carte 260, moustache vs. moustasse) :

«Les branches de bayahondes les fouettaient au visage, des débris de chandelles, de cadasses et d'autres cactées leur piquèrent les pieds. » J.-St. Alexis, Compère Général Soleil, Paris, Gallimard, 1955, p. 328. 
«Le soleil couchant rendait plus sombres les grands cadasses tendant leurs branches nues droit vers le ciel, telles des bouquets de cierges. » Id., ibid., p. 330.

«Le sentier se perdit subitement dans une haie touffue qui s'élevait à trois mètres du sol, aloès, cadasses et autres plantes épineuses, ainsi que des lianes rampantes qui envoyaient des rejets, des bouquets d'orchidées, roses, mauves ou tachetées de sang. » S. Schwarz-Bart, Ti Jean L'horizon, Paris, Seuil, 1979, p. 44.

Quoi qu'il en soit, le mot a été repris comme tel par le traducteur, créant encore une fois un néologisme artificiel qui ne pourrait pas être compris sans une note de bas de page (laquelle prétend que le mot veut dire « enebro », c'est-à-dire « genévriers », un arbre aux feuilles acérées ; c'est ce que l'on peut imaginer à partir du contexte, mais ce n'est pas nécessairement la même plante). Notons que le texte original ne comportait pas de note de bas de page, le contexte renseignant plus ou moins le lecteur exogène sur la nature du référent.

On constate aussi que la lexicographie des créoles n'est jamais complète : on ne trouve nulle part le type kadas pour désigner la plante (malgré J.-St. Alexis et S. Schwarz-Bart).

Ascencio $(2004,152)$ traduit par tuna, mot qui désigne le nopal, plante de forme et de fonctions similaires (v. DRAE 2001).

BILAN BIBL. : ø Friederici 1947 ; ø Pompilus 1961 ; ø Arveiller 1963 ; ø TLF ; « cadastre » Tourneux / Barbotin 1990 s.v. kadas ; ø Telchid 1997 ; ø DRAE 2001 ; ø Ludwig et al. 2002; «cadastre» Confiant 2007 s.v. kadas ; «[land] deed» Valdman 2007 s.v. kadach ${ }^{1}$; « kind of cactus » Valdman 2007 s.v. kadach 2.

calebassier n. m. "arbre d'Amérique appartenant à la famille des Bignoniacées" (TLF).

«Bienaimé, son mari, fume sa pipe, la chaise calée contre le tronc d'un calebassier. » (p. 267)

«Bienaimé, su marido, fuma la pipa, con la silla apuntalada contra el tronco de un calabacero. » (p. 2)

Note de bas de page : «Árbol de Centroamérica. »

- Le mot est bien répertorié dans le TLF. Quant au mot espagnol donné ici en guise de traduction, calabacero, il figure dans le DRAE 2001 mais désigne un autre référent, à savoir la plante potagère qui donne les courges (« Planta anual de la familia de las Cucurbitáceas, con tallos rastreros muy largos y cubiertos de pelo áspero, hojas anchas y lobuladas y flores amarillas. Su fruto es la calabaza. »). Selon le Grand Dictionnaire espagnol- 
français / français-espagnol des éditions Larousse-Bordas (1998), ce qui s'appelle calebasse en français est rendu dans les diverses variétés d'espagnol américain par de nombreuses appellations : calabaza, güira, tapara, cujete, jicara, mate, totuma. Quant au nom désignant la plante, calebassier, le Larousse bilingue le rend par taparo (c'est le choix de traduction d'Ascencio 2004, 121; le DRAE 2001 le présente comme propre au Vénézuéla) ou güira. La note de bas de page attire l'attention du lecteur sur le fait qu'il ne s'agit pas du calabacero tel qu'on l'entend en Espagne, mais ne nous donne pas un vrai équivalent en espagnol d'Amérique, ni du reste une définition suffisamment précise.

BILAN BIBL. : TLF ; LarBil 1998 ; DRAE 2001.

caraban n. m. "piège à oiseaux".

«Et si moi, je voulais parler aussi ? Je dirais : qui est-ce qui lui a appris à manier la houe et la serpette, à sarcler, à planter, et même à faire des carabans pour attraper les oiseaux ?» (pp. 328-329)

Note de bas de page (de Jacques Roumain) : « Pièges. »

« ¿Y si yo quisiera hablar también? Yo diría : ¿Quién le ha enseñado a manejar la azada y la podadera, a escardar, a plantar y aún a hacer caravanas para atrapar pájaros?» (p. 118)

Note de bas de page : «Término empleado en Cuba aplicado a la trampa para cazar pájaros. »

- Il s'agit fort probablement d'un autre emprunt du créole haïtien (qui apparaît chez Roumain sous une forme francisée) à un mot espagnol, plus précisément à une acception cubaine de ce mot, s'il faut en croire Corominas : « Por otra parte, correr la caravana tomaría el significado de 'hacer algo peligroso' (como un ataque marítimo), de ahí la ac. argentina 'correr la tuna' (Segovia), así como el ecuat. carabana 'peripecia, accidente, contratiempo' [...] y el significado cubano 'trampa a manera de jaula en que se hace caer a las aves' [...]. ». Le traducteur a bien vu que le mot cubain était apparenté au mot haïtien, dans sa forme et dans son sens ; sa traduction est très justifiée, mais la note de bas de page est tout de même bienvenue pour éclairer un public hispanophone exogène.

La traduction d'Ascencio $(2004,188)$ est résolument cibliste, avec l'équivalent diatopiquement neutre trampas.

BILAN BIBL. : ø Pompilus 1961 ; DCECH 1, 855b s.v. caravana; «Cuba [...] trampa para pájaros » DRAE 2001 s.v. caravana 6 ; «bird-trap » Valdman 2007 s.v. karabann (kabann). 
cassave n. f. "racine de manioc; (par méton.) fécule extraite de cette racine ; (par méton.) galette faite de cette fécule" (déf. adapté du TLF).

« Il trempait un morceau de cassave dans son café. » (p. 292)

« Hundía un trozo de casabe en su café. » (p. 49)

Note de bas de page : «Torta o galleta de harina de la raíz de la yuca. »

- Type lexical abondamment documenté depuis l'époque coloniale; le français l'a emprunté à l'espagnol, qui lui le tient du taino (arawak de Haïti). Comme le précise le TLF, «[1]e mot a été vulgarisé par l'intermédiaire du 'baragouin' parlé par les indigènes des petites Antilles, mélange de caraïbe et d'esp. ». Du français colonial, il est passé au créole.

Comme le mot français vient du mot espagnol, il n'y avait pas vraiment de problème de traduction; on se demande même à quoi sert la note de bas de page (mais il est vrai que le référent n'est pas connu dans tout le monde hispanophone, car il s'agit plutôt d'une réalité antillaise). En espagnol, le mot existe sous deux graphies : cazabe et casabe (v. DRAE 2001). Le traducteur a retenu la variante avec $-s-$, qui en effet reproduit mieux la prononciation de l'espagnol seseísta du Nouveau Monde. C'est aussi le choix d'Ascencio $(2004,149)$.

BILAN BIBL. : Friederici 1947 s.v. cazabi ; TLF ; Fattier 1998, t. 1, p. 425 et carte 951 ; DRAE 2001 ; « cassava flatbread 》 Valdman 2007 s.v. kasav (kasab).

chandelier n. m. "(espèce de cactus)".

«Une bande de corbeaux s'abat sur les chandeliers.» (p. 267)

«Una bandada de cuervos se abate sobre los candelabros. » (p. 2)

Note de bas de page : «Árbol de Centroamérica. »

- Ce type lexical n'a été attesté dans la lexicographie jusqu'à maintenant que dans Valdman 2007, sous sa forme créole ; l'attestation chez Roumain en représente la version francisée. Alors que le TLF ne donne rien de satisfaisant du point de vue sémantique s.v. chandelier, on trouve en revanche l'acception suivante s.v. candélabre B 2: «ARBORIC. Arbre ou arbuste dont la forme évoque celle d'un candélabre». Cet emploi existe également en créole haïtien, v. Valdman 2007 s.v. kandelab et Faine 1974 s.v. candélabre. Ce dernier précise : «Un cactus très épineux qui sert à faire des haies porte le nom de 'candélabe' à cause de sa forme et de la 
disposition de ses branches. » On peut donc considérer que la traduction par esp. candelabro est tout à fait appropriée, à plus forte raison si l'on considère que ce mot est déjà dans le DRAE 2001 : «planta de la familia de las Cactáceas, cuyos frutos se llaman tunas, peladas, o chulas. Alcanza una altura de más de seis metros y se cría en varias provincias de Argentina y en México.»

La traduction d'Ascencio $(2004,121)$ propose également comme équivalent candelabro.

BILAN BIBL. : Ø Friederici 1947 ; ø Pompilus 1961 ; ø Arveiller 1963 ; DRAE $2001 ;$ « 4 kind of cactus ef kandelab ${ }^{7} »$ Valdman 2007 s.v. chandelye.

clairin n. m. "eau-de-vie obtenue par la fermentation du jus de canne".

« Merci, maman. C'est un bon clairin et bien réchauffant. » (p. 284)

Note de bas de page (de Jacques Roumain) : « Alcool de canne à sucre. »

« Gracias, mamá. Es un buen clerén y muy reconfortante.» (p. 34)

Note de bas de page: «La palabra clairin ha pasado fonéticamente a Santo Domingo por clerén, aguardiente de caña.»

- Type lexical haïtien d'origine incertaine ; il pourrait s'agir d'un dérivé formé sur l'adj. clair évoquant la couleur de cette boisson (Valdman précise bien " unrefined white rum »). On trouve dans FEW 2, 740ab, CLARUS I 1 de nombreux dérivés de clair désignant divers types de boissons alcoolisées.

Le traducteur a opté pour le mot de l'espagnol dominicain qui représente un emprunt direct au mot français. C'est une très bonne solution, mais la note de bas de page est nécessaire car un lecteur hispanophone exogène ne peut pas deviner de quoi il s'agit. On aimerait bien, en outre, avoir une référence bibliographique (nous n'avons pas retrouvé le mot dans les sources à notre disposition, mais c'est vrai qu'il abonde dans GRL, en référence à la République Dominicaine; on le trouve aussi une fois dans CORDE, dans un recueil de « haitianismos / dominicanismos »).

Ascencio $(2004,140)$ traduit également par clerén.

BILAN BIBL.: Pompilus 1961, 152; «raw rum, cheap unrefined white rum » Valdman 2007 s.v. kleren.

${ }^{7}$ Cf. « 2 candelabra cactus » Valdman 2007 s.v. kandelab. 
coumbite n. m. "travail agricole collectif".

« À l'époque, on vivait tous en bonne harmonie, unis comme les doigts de la main et le coumbite réunissait le voisinage pour la récolte ou le défrichage. » (p. 269)

Note de bas de page (de Jacques Roumain) : «Travail agricole collectif. »

« En aquel entonces, vivían todos en buena armonía, unidos como los dedos de la mano y el cumbite reunía al vecindario para la cosecha o el desmonte. » (p. 5)

Note de bas de page : « Trabajo agrícola colectivo. Se atribuye su origen a la palabra 'convite'. »

- Selon Pompilus (suivi par Faine), viendrait «[d]e l'espagnol convite [sic sans italiques]». Ce mot espagnol signifie au sens propre «invitation », mais il a surtout désigné par le passé un grand repas de banquet (« comida o banquete a que es convidado alguien » DRAE 2001); à partir de ce sens, par métonymie, s'est développé le sens de « reunión de trabajadores que prestan sus servicios a cambio de comida», donné comme propre au Vénézuéla par le DRAE 2001 (sens confirmé par le Diccionario del habla actual de Venezuela), mais certainement connu ailleurs, car c'est avec ce dernier sens que le mot a été emprunté en créole haïtien. Le traducteur a repris le mot tel quel, avec sa graphie créole (à l'exception d'une unique concession à l'orthographe de l'espagnol selon laquelle le son $[\mathrm{u}]$ est rendu dans cette langue par le graphème $\langle\mathrm{u}\rangle$ et non par le digramme «ou〉 comme en français), plutôt que de l'écrire convite, alors que le mot espagnol est pourtant bien attesté avec ce sens. Au moins, une note de bas de page renseigne le lecteur sur l'origine espagnole du mot créole.

Ascencio $(2004,123)$ traduit également coumbite par cumbite plutôt que par convite. Dans les deux cas, les traducteurs ont préféré maintenir autant que possible la sonorité du mot créole, plutôt que de le rendre par le mot espagnol qui en est l'étymon.

BILAN BIBL. : Pompilus 1961, 56, 163 (qui relève les graphies combite ou coumbite); "Le 'coumbite' est une sorte de corvée coopérative où les membres de la 'société' s'engagent volontairement à fournir la maind'œuvre en vue de certains travaux des champs. [...] Etym. - Coumbite, doublet de 'convite', mot esp.» Faine 1974 s.v. corvée ; DRAE 2001; « collective rural work, cooperative peasant work team for clearing land and harvesting, etc. » Valdman 2007 s.v. konbit (koumbit, kounbit). 
don n. m. "grand propriétaire terrien".

« C'était un véritable don, si tu as mémoire, ce général Lonjeannis, un nègre de grandes manières, un patriarche : on n'en voit plus de ce format. » (p. 299)

Note de bas de page (de Jacques Roumain) : «Gros propriétaire paysan. Vient de l'espagnol. »

«Era un verdadero Don, ese general Lonjeannis, si haces memorias, un negro de grandes maneras, un patriarca : no se ven más de ese tipo. » (p. 63)

Note de bas de page : «Palabra española empleada en Haití para designar al rico propietario campesino.»

- Emprunt à l'espagnol, avec restriction sémantique : le mot en espagnol s'utilise devant le prénom de n'importe quel homme à qui on veut témoigner du respect, alors qu'ici l'emploi est restreint à un grand propriétaire terrien, en contexte rural. Il est normal que le mot soit conservé comme tel dans la traduction, mais la note de bas de page est nécessaire pour que le lecteur hispanophone comprenne cet emploi particulier du mot en Haïti.

Ascencio $(2004,157)$ repend don mais sans la majuscule, qui est en effet inhabituelle en espagnol avec ce mot; une note est également présente pour désambiguïser ce faux-ami.

BILAN BIBL. : «mot d'origine espagnole probablement » Pompilus 1961, 163-164 (avec deux citations, dont celle de Roumain) ; «titre de courtoisie $[\ldots]$ On n'emploie guère plus ce mot que pour désigner les campagnards plus ou moins aisés ou influents; on le fait précéder le plus souvent par l'adj. grand» Faine 1974 s.v. don; « Tratamiento de respeto, hoy muy generalizado, que se antepone a los nombres masculinos de pila. Antiguamente estaba reservado a determinadas personas de elevado rango social. » DRAE 2001 s.v. don ; «large landowner, big landowner; person of importance [in rural area] » Valdman 2007 s.v. grandon (don).

farinade n. f. "légère pluie qui semble tomber comme de la farine".

" Autrefois en cette saison, dès le matin, le ciel se mettait à la grisaille, les nuages s'assemblaient, gonflés de pluie, pas une grosse pluie, non, tout juste, quand les nuages crevaient comme des sacs trop pleins, une petite farinade, mais persistante, avec quelques éclaircies de soleil. » (p. 274)

«Antes, en esta época, desde la madrugada, el cielo se ponía grisáceo, las nubes se reunían, hinchadas de lluvia, no una gran lluvia, no, cuando 
las nubes reventaban como bolsas demasiado llenas, justo una pequeña jarina, pero persistente, con alguna clareza de sol. » (p. 14)

Note de bas de page: «La palabra es farinade que encuentra su equivalente en el vocablo dominicano jarina que significa una lluvia fina o llovizna. »

- Innovation formelle créée par dérivation sur farine à l'aide du suffixe -ade exprimant une quantité ; correspond au créole haïtien farinay. S'il est bien vrai que l'espagnol dominicain connait l'équivalent jarina pour désigner le même référent, il s'agit alors d'une bonne trouvaille (car l'étymon est le même ; jarina représente la forme espagnole harina avec maintien de l'ancien $h$ - aspiré, qui remonte au latin FARINA, tout comme le mot français farine). Nous avons en effet trouvé jarina «llovizna » dans le Diccionario ejemplificado de canarismos (Corrales / Corbella 2009) qui l'atteste pour l'île de Higuera. On trouve aussi le diminutif harinita avec le même sens dans le Diccionario Histórico del español de Canarias (Corales / Corbella 2001), ainsi que le verbe harinar « lloviznar », parallèlement à celui que l'on trouve en français régional haïtien (v. ci-dessous, citation de Pompilus) ainsi qu'en créole (v. Valdman, bibl. ci-dessous).

Ascencio $(2004,129)$ traduit par lloviznita pequeña (littéralement, «toute petite pluie »), un choix cibliste qui neutralise le particularisme diatopique.

BILAN BIBL. : «La pluie farine, est une autre expression imagée du F. H. pour désigner la chute d'une pluie fine, qui rappelle de la farine jetée au vent. » Pompilus 1961, 141 ; «drizzle, sprinkle [rain], light rain » Valdman 2007 s.v. farinay; « to drizzle, sprinkle [rain], rain lightly » Valdman 2007 s.v. farinen.

flamboyant n. m. "arbre tropical à fleurs rouges" (TLF).

« Et le feuillage déchiqueté des arbres à pain, rapiécé d'azur, et le feu du flamboyant longtemps couvé sous la cendre de la nuit et qui, maintenant, éclate en un boucan de pétales à la lisière des bayahondes. » (p. 270)

«Y el follaje recortado de los árboles de pan, remendados de azul, y el fuego del flamboyán largo tiempo escondido en las cenizas de la noche, y que estalla, ahora, en un estrépito de pétalos, a orilla de las bayahondas. » (p. 8)

Note de bas de page : «Una especie de tulipán pequeño. »

- Comme le dit avec raison le Grand Robert 2008, ce « mot, didactique ou littéraire en Europe, est courant en Afrique francophone et aux Antilles. » 
(cas typique de régionalisme de statut). Mais il désigne un arbre de taille respectable ${ }^{8}$, alors que la note de bas de page de la traduction prétend qu'il s'agit d'une "espèce de petite tulipe»! Le mot espagnol flamboyán (adaptation évidente du français flamboyant) est répertorié dans le DRAE 2001 avec la définition suivante : «Cuba, Guat. y Méx. Árbol de la familia de las Leguminosas, oriundo de la India, que en el verano echa flores de color rojo anaranjado en ramillete. » $C$ 'est toutefois la première édition du DRAE dans laquelle le mot figure; le traducteur n'avait vraisemblablement à sa disposition aucun dictionnaire, français ou espagnol, qui eût pu le renseigner sur le sens du mot. Du coup, il aurait mieux fait de ne pas mettre de note du tout. Mais il devait toutefois être familier avec la forme graphique flamboyán, car elle est très bien attestée dans GRL (pages en espagnol), dans des livres antérieurs à la date de la traduction, malgré son apparition très tardive dans le DRAE.

La traduction d'Ascencio $(2004,125)$ maintient exactement la même forme graphique que le français, flamboyant, alors que des adaptations graphiques sont disponibles dans la lexicographie espagnole.

BILAN BIBL. : TLF ; DRAE 2001 ; « flamboyant, flame tree, royal poinciana » Valdman 2007 s.v. flambwayan; GR 2008.

frangipanier n. m. "arbrisseau, originaire de l'Amérique tropicale, dont les fleurs ont un parfum rappelant celui de la frangipane" (TLF).

« Non, il n’y avait ni justice ni raison dans cette histoire. Il fallait laisser les morts reposer dans la paix du cimetière sous les frangipaniers. » (p. 313)

«No, no había ni justicia ni razón en esta historia. Era preciso dejar reposar a los muertos en la paz del cementerio, bajo los frangipanes. » (p. 90)

Note de bas de page : « Arbusto de América parecido al laurel rojo. »

- Mot bien répertorié dans la lexicographie française (v. TLF) ; le LarBil 1998 propose de la traduire par amancayo ou súchil. Le premier de ces mots est défini comme suit dans le DRAE 2001 : «Am. Nombre de diversas plantas, ya herbáceas, ya arboráceas, cuya flor, blanca o amarilla, recuerda a la azucena. ». C'est donc un équivalent passe-partout. Voici la définition que le second de ces mots a reçu dans la même source : "Méx. p. us. flor (\| brote de las plantas, formado por hojas de colores) ». Cette

${ }^{8}$ « Árbol, oriundo de Madagascar, que alcanza hasta $12 \mathrm{~m}$ de altura, con un tronco de hasta $90 \mathrm{~cm}$ de diámetro. " Diccionario del español de Cuba, G. Cárdenas Molina, A. Ma Tristá Pérez, R. Werner, Madrid, Gredos, 2000. 
définition ne correspond pas à ce que donne le LarBil (qui donne frangipanier comme équivalent français de súchil). En cherchant dans le Diccionario del español de América de Morínigo (1993), on trouve pas moins de quatre acceptions pour ce mot: «Gua. Cierta bebida compuesta que se toma como refresco. 2. Méx. Ramillete de flores vistosas y fragrantes. 3. Final de una fiesta nocturna. 4. Magnolia. » Enfin, dans le Diccionario de regionalismos de la lengua española de Grosschmid et Echegoyen (1998), le mot est défini comme suit : « Méjico Árbol apocináceo pequeño, de buena madera. » La solution la plus sage serait peut-être d'opter pour amancayo, suffisamment vague pour ne pas être faux.

Quoi qu'il en soit, le traducteur a été un peu paresseux en choisissant de rendre le mot par frangipanes, forme qui en espagnol n'existe pas, mais qui donne l'impression d'être l'adaptation de frangipane plutôt que de frangipanier. Quant à Ascencio (2004, 172), elle traduit par amapola, mot qui en Espagne désigne normalement le coquelicot, mais qui en Amérique latine est le «[n]ombre de varias plantas americanas de diversas familias, semejantes en algún aspecto a la amapola común » (DRAE 2001); encore une fois, c'est l'imprécision référentielle qui permet cet emploi.

BILAN BIBL.: TLF ; Morínigo 1993; Grosschmid / Echegoyen 1998 ; LarBil 1998 ; « frangipani » Valdman 2007 s.v. franjipànye.

gaguière n. f. "lieu où se tiennent les combats de coqs".

« Est-ce que tu viendras demain-si-dieu-veut à la gaguière? ? (p. 297)

Note de bas de page (de Jacques Roumain) : «Où ont lieu les combats de coq. "

«- ¿Vendrás mañana, si Dios quiere, a la gallera ?» (p. 58)

Note de bas de page : « Donde tienen lugar las riñas de gallos. »

- Comme l'explique très bien Pompilus (bien qu'avec une typographie catastrophique), «[1]e mot vient de l'espagnol Gallera sorti lui-même de 'gallo, coq'; 1 mouillé a évolué en ibéro-américain en y, puis dj, puis g (cf. créole haïtien, gadyè, gadjè et gagê). » Il s'agit là de l'un des nombreux mots haïtiens empruntés à la langue espagnole. Dans les Petites Antilles, pour exprimer le même concept, on dit pit (de l'anglais) ou gallodrome (formation savante), v. Thibault 2009, 125-126.

Le mot, venant de l'espagnol, était très facile à traduire dans cette langue; toutefois, la note de bas de page qui était nécessaire en français devient tout à fait superflue dans le texte d'arrivée et on ne comprend pas bien pourquoi elle a été maintenue. Ascencio $(2004,154)$ s'en passe d'ailleurs très bien. 
BILAN BIBL. : « combat de coqs; lieu destiné à des combats de coqs » Pompilus 1961, 175-176 s.v. gagaire, gaguère; Fattier 1998, t. 2, p. 560 et carte 1271 ; «gallinero en que se crían los gallos de pelea; edificio construido expresamente para las riñas de gallos; jaula donde se transportan los galos de pelea » DRAE 2001; « arena [cockfight]» Valdman 2007 s.v. gagè (gadjè, gadyè).

gros-bleu n. m. "type de tissu en coton ou en lin, très résistant, de couleur bleue, dans lequel on fait des vêtements de travail". Cf. français de référence coutil, denim, jean; français des Petites Antilles drill.

«C'est que les choses prenaient mauvais visage, la faim se faisait sentir pour tout de bon, le prix du gros-bleu montait en ville, alors on avait beau raccommoder le linge, il y en avait dont le derrière, sauf votre respect, paraissait par les bâillements du pantalon comme un quartier de lune noire dans les déchirures d'un nuage, ce qui n'était pas honorable, non, on ne pouvait pas le prétendre. » (p. 311)

«Es que las cosas tomaban mal cariz, el hambre se hacía sentir seriamente, el precio del gros-bleu subía en la ciudad, entonces, en vano se remendaba la ropa, había alguno cuyo trasero, con perdón de usted, aparecía por las roturas del pantalón como un cuarto de luna negra entre los desgarrones de una nube, lo que no era honorable, no, no se podía pretenderlo. » (p. 85)

Note de bas de page : «Tejido de algodón de color azul, con el que confeccionan sus vestimentas las capas pobres de la población haitiana. ( $N$. del E.).»

REM. : En français de référence, gros-bleu se dit d'un «vin de médiocre qualité, âpre et peu corsé » (TLF).

- Cet emploi est bien sûr à rattacher au fr. de réf. bleu n. m. "combinaison de travail" (cf. les lexies composées bleu de travail, bleu de mécanicien, être en bleu, tous TLF s.v. bleu D 2). C'est le même procédé qui est à l'œuvre, mais la métonymie s'applique ici de la couleur au tissu, et non au vêtement dont il est fait.

Le traducteur aurait pu traduire par l'esp. dril (« tela fuerte de hilo o de algodón crudos » DRAE 2001), mot du reste bien attesté dans le français régional des Petites Antilles, mais l'emprunt cru permet d'injecter un peu de « couleur» locale. Ascencio $(2004,170)$ a aussi maintenu la forme française, mais en l'affublant d'une faute d'orthographe (*gros-bleue).

BILAN BIBL. : ø Pompilus 1961 ; «gwo ble denim type of fabric» Valdman 2007 s.v. ble 2 . 
herbe de Guinée loc. subst. f. "graminée répandue aux Antilles, pouvant servir de fourrage, mais considérée comme parasite dans les plantations de canne à sucre" (Thibault 2008, 269-270).

«On entrait dans l'herbe de Guinée! (Les pieds nus dans la rosée, le ciel pâli, la fraîcheur, le carillon des pintades sauvages au loin...)» (p. 269)

« ¡Entraban en la hierba de Guinea! (Los pies desnudos dentro del rocío, el cielo pálido, el frescor, el griterío de las gallinas salvajes a lo lejos...)»(p. 6)

Note de bas de page : «Planta gramínea muy usada en África para alimentar el ganado. »

- $1^{\text {re }}$ attestation : 1797 ( « Les fourrages qui servent de nourriture des bestiaux sont le bois-patate, c'est-à-dire, la tige et les feuilles de cette racine ; celle du petit mil en vert, l'herbe de Guinée » Wimpffen, 138 ; v. Rézeau 2008 s.v. bois-patate). Innovation par composition : subst. herbe + complément prépositionnel introduit par de exprimant l'origine. Le type créole est parfois défini par «herbe coupante» dans les sources antillaises (v. bibl. ci-dessous) ; il s'agit probablement d'un régionalisme caché du français régional antillais dans la métalangue. - On retrouve la même lexie en français régional de Nouvelle-Calédonie : v. Pauleau $2007^{1,2}$ s.v. herbe de Guinée (Panicum maximum).

Le traducteur a rendu la lexie par son équivalent espagnol, qui y correspond mot à mot, sans être pour autant un artefact : on trouve bel et bien plusieurs centaines d'attestations de la lexie composée hierba de Guinea dans GRL, et très souvent en référence à Cuba et à la République Dominicaine ; en outre, elle se trouve même dans le DRAE 2001, avec la définition suivante : «planta de la familia de las Gramíneas, que crece hasta cerca de un metro de altura, con hojas ensiformes, radicales, abrazadoras y en macolla, tallo central, y flores hermafroditas, en espiguilla, que forman panoja, con semillas abundantes. Es planta muy apreciada para pasto del ganado, especialmente caballar, y se propaga con facilidad en las regiones tropicales. » La note prétend que cette herbe serait «très utilisée en Afrique pour nourrir le bétail », alors que d'après la plupart des sources retrouvées dans GRL, autant pour le français que pour l'espagnol, c'est bien justement dans les Antilles que cette herbe (ainsi que le terme qui la désigne) est très connue.

Ascencio $(2004,124)$ traduit également par hierba de Guinea.

BILAN BIBL.: "zhèbe guinẽe, panicum maximum (JACQ.) = graminée introduite aux Antilles » Jourdain 1956, 290 ; DRAE 2001 s.v. hierba; « herbe coupante (Panicum maximum)» Ludwig et al. 2002 s.v. giné; 
《zèb-diné/zéb giné : Herbe coupante » id. s.v. zèb ; « herbe de Guinée » Confiant 2007 s.v. zeb-djinen; « zèb ginen guinea grass 》 Valdman 2007 s.v. $z \grave{e} b^{1}$; Thibault 2008, 269-270.

jouquer v. intr. "se percher, se poser, se jucher".

«C'est là que les ramiers ont jouqué. » (p. 335)

« Es allí que [sic; donde] las torcazas han yuqueado.» (p. 131)

Note de bas de page: «Jouqué en el original. En Perú se emplea yuquear por buscar la yuca silvestre que crece en despoblado : La yuca es planta que crece también en las Antillas y es alimento del campesino pobre. »

- Le mot créole est tout simplement un héritage d'un verbe attesté dans de très nombreux parlers galloromans, entre autres dans le Grand Ouest et au Canada (v. FEW) ; le verbe concurrent qui a triomphé en français standard, jucher, est né d'un croisement entre jouquer et hucher, de même sens.

Le traducteur n'a pas compris le sens du mot. Il propose, sans doute pour des raisons de paronymie, de traduire par le péruvianisme yuquear, un dérivé de yuca qui n'a absolument rien à voir sémantiquement avec jouquer. Ascencio (2004, 195 et note 59) traduit plus simplement (et plus correctement) par se posaron.

BILAN BIBL. : FEW 16, 288a, JŬK 1 ; ø Pompilus 1961 ; « $v$ intr. to roost, perch » Valdman 2007 s.v. jouke ${ }^{l}$ II.

latanier n. m. "palmier à grandes feuilles textiles" (TLF).

«Il prit le chemin d'une butte couronnée de lataniers." (p. 278)

«Tomó el camino de un cerrillo coronado de lataneros." (p. 22)

Note de bas de page : « Palmera grande.»

- Mot bien représenté dans la lexicographie française, att. dp. 1645, d'origine caraïbe (v. TLF). Bien que le LarBil 1998 propose l'espagnol latania comme équivalent (lequel est effectivement attesté dp. 1899 dans le DRAE), il faut dire que l'équivalent latanero est assez bien représenté dans GRL (pages en espagnol) ; mais il doit s'agir d'un calque morphologique formé sur le mot français. 
Ascencio $(2004,134)$ propose un équivalent cibliste et hypéronymique, palmas.

BILAN BIBL. : DRAE 1899 à 2001 (pour latania); TLF ; LarBil 1998.

loa n. m. "divinité afro-haïtienne".

«On prie pour la pluie, on prie pour la récolte, on dit les oraisons des saints et des loa. » (p. 293)

Note de bas de page (de Jacques Roumain) : «Divinités afro-haïtiennes. »

«Se reza por la lluvia, se reza por la cosecha, se dicen las oraciones de los santos y de las loa. » (p. 51)

Note de bas de page : « Divinidades afrohaitianas. »

- Type lexical d'origine incertaine. Il a été repris tel quel par le traducteur, car il renvoie en effet à un référent qui ne peut être appelé autrement (à moins d'user d'une périphrase ou d'un équivalent approximatif). On remarque que le mot, masculin en français régional antillais, est devenu féminin dans cette version. Le traducteur ignorait probablement le genre du mot, mais l'a traité comme féminin peut-être en raison du - $a$ final et du fait que le mot désigne des divinidades, c'est-à-dire des déités, mot féminin. On remarque aussi, ce qui est encore plus surprenant, que le mot a été traité comme invariable (en nombre) en espagnol, à l'imitation de ce que l'on observe en français : le $-s$ du pluriel, qui est pratiquement obligatoire en espagnol, est absent. Cela s'explique en français en raison de l'origine créole du mot (il n'y a évidemment pas de $-s$ de pluriel en créole !), et est facilité par le fait que le $-s$ de pluriel du français ne se prononce pas de toute façon. En espagnol, cette particularité morphologique choque davantage. D'ailleurs, Ascencio (2004, 150 et note 28) opte pour «los loas », au masculin et avec le $-s$ du pluriel.

BILAN BIBL. : Pompilus 1961, 161-162 ; DUF 1997 ; Fattier 1998, II, 589590 et carte 1342 ; Hénane 2004 s.v. loa ; « voodoo deity » Valdman 2007 s.v. lwa; Zanoaga 2011.

macoute n. f. "grand sac en matière textile".

«Voici ta macoute, avec les vivres dont tu auras besoin dans ton voyage de retour. Rien ne manque : l'épi de maïs boucané, arrosé de sirop et d'huile d'olive, des salaisons, des gâteaux et la liqueur pour ta soif. » (p. 303) 
«He aquí tu macuto con los víveres que necesitarás en tu viaje de regreso. No falta nada : la espiga de maíz ahumado regada de almíbar y aceite de oliva, salazones, tortas y licor para tu sed. » (pp. 69-70)

Note de bas de page: «En Cuba, Santo Domingo y Venezuela la mochila de limosnero. En Cuba es también un paquetito hecho de yagua que contiene cera, carne o tabaco, etc. »

- Type lexical d'origine africaine (v. Bollée 2011, 38-39), bien répandu dans les pays hispanophones de l'aire caraïbe, probablement passé de là aux créoles et au français des Antilles.

Le traducteur n'a eu aucune peine à rendre le mot par son équivalent espagnol, tout en l'accompagnant d'une note de bas de page, bien que celle-ci soit beaucoup moins nécessaire en espagnol qu'en français (le mot est dans le DRAE 2011). La traduction d'Ascencio (2004, 160), mochila, est en revanche encore une fois résolument cibliste.

En Haïti, le mot est entré dans la formation d'une lexie composée, tonton macoute, qui désignait à l'origine un personnage de croquemitaine ; puis, de là, le mot a été employé pour se référer aux hommes de main du régime de Duvalier.

BILAN BIBL. : ø Friederici 1947 ; «récipient en fibre de latanier, de grandeur variable, servant à divers usages domestiques [...] De l'espagnol macuto 'panier de mendiant' et, dans le langage militaire 'sac' » Pompilus 1961, 57, 150; ø Arveiller 1963 ; «Macoute ou Macaouti, mots ind.car. » Faine 1974 s.v. besace ; « macuto, cub., domin., venez., 'saco largo y estrecho que llevan al hombro los jornaleros, soldados, etc.', origen incierto, quizá negro africano. $1 .^{a}$ doc. : 1836, Pichardo. » DCECH 3, 745b ; DECOI II, 1993, s.v. makuti; " (Voz de las Antillas y Venezuela). m. Mochila, especialmente la del soldado. || 2. Ven. Cesto tejido de caña amarga, de forma cilíndrica y con asa en la boca, que se usa para transportar víveres y otros objetos. » DRAE 2001 s.v. macuto ; «à l'origine : sac. Aujourd'hui : le sein, contre la poitrine, sous la chemise » Ludwig et al. 2002 s.v. makout; " straw or matted grass bag with shoulder strap (usually carried by a man); saddlebag » Valdman 2007 s.v. makout ${ }^{l}$; « thug that served as militia during the Duvalier regime» Valdman 2007 s.v. makout ${ }^{2}$; «folk character [bogeyman] ; member of the Duvalierist militia » Valdman 2007 s.v. tonton ${ }^{2}$; « (arch.) sac» Confiant 2007 s.v. makout 1; Bollée 2011, 38-39.

madras n. m. "étoffe à chaîne de soie et à trame de coton, de couleurs vives ; (p. méton.) pièce de cette étoffe utilisée comme foulard, fichu, cravate ou mouchoir" (TLF). 
«La poussière monte de la grand'route et la vieille Délira est accroupie devant sa case, elle ne lève pas les yeux, elle remue la tête doucement, son madras a glissé de côté et on voit une mèche grise saupoudrée, dirait-on, de cette même poussière qui coule entre ses doigts comme un chapelet de misère [...]. » (p. 267)

« La polvareda sube de la carretera y la vieja Délira está acurrucada ante su choza, no levanta los ojos, mueve la cabeza dulcemente, su madrás se ha deslizado a un costado y asoma una mecha gris que se diría espolvoreada con ese mismo polvo que resbala entre sus dedos como un rosario de miserias [...]. » (p. 1-2).

Note de bas de page : «Tela de algodón. En Cuba se denomina así a un pañuelo de hilo del tipo de madrás, pañuelo bayajá. En Santo Domingo se le da el nombre de fulá, evidentemente vocablo de origen francés (foulard), al pañuelo usado por las ancianas en la cabeza y al turbante que usan las haitianas. »

- Régionalisme de fréquence des variétés de français antillaises et de l'Océan Indien (cas typique de «mot des Isles », diffusé à l'époque coloniale; de Madras, nom d'une ville de l'Inde où cette étoffe était fabriquée). Le mot espagnol madrás apparaît déjà dans le DRAE 2001, mais seulement avec l'acception première « tejido fino de algodón que se usa para camisas y trajes femeninos ». Il était relativement facile de l'utiliser avec le sens métonymique qu'on lui trouve dans le texte de départ.

Malgré ce que l'on peut lire dans la note (et qui n'est pas clair : veut-on nous dire qu'à Cuba le foulard en question s'appelle madrás, ou pañuelo bayajá ?), le traducteur n'a pas osé utiliser le gallicisme fulá propre à l'espagnol de Santo Domingo pour rendre madrás, et a préféré s'en tenir à une forme déjà connue en espagnol qui a justement la même origine que le mot utilisé dans le texte de départ (bien qu'avec une acception légèrement différente). Quant à madrás au sens de "foulard" et (pañuelo) bayajá, ils sont absents de Cárdenas Molina / Tristá Pérez / Werner (coord.) 2000, tout comme de Richard (coord.) 2000.

La traduction d'Ascencio $(2004,121)$ a recours à un mot diatopiquement neutre, pañoleta.

BILAN BIBL. : TLF ; DRAE 2001.

mal caduc loc. nom. m. "épilepsie".

« Et maintenant, ce Manuel qui se conduisait comme s'il allait tomber du mal caduc, quand donc, par la barbe du Saint Esprit, pardon, mon Dieu, j'ai blasphémé, je ne le ferai plus, mea culpa, quand donc finiraient tous ces emmerdements ?» (p. 338) 
«Y, ahora, ese Manuel que se comportaba como si fuera a caer con el mal caduco, cuando pues, por las barbas del Espíritu Santo -mea culpa-, perdón, Dios mío, he blasfemado, no lo haré más, ¿cuándo, pues, acabarían todas estas tribulaciones? » (p. 136)

Note de bas de page : «Voz popular empleada en Haití para designar la epilepsia. »

- En fait, ce mot a appartenu très longtemps au français populaire nonmarqué régionalement, comme on peut le constater en consultant l'article caduc du TLF, qui donne mal caduc «épilepsie» comme «vx. », tout simplement. À vrai dire, l'équivalent espagnol donné par le traducteur, mal caduco, appartient lui aussi à l'espagnol général (il apparaît dans le DRAE 2001 sans aucune marque de restriction diasystémique). La traduction est tout à fait correcte, mais la note de bas de page semble assez superflue. Quant à la traduction d'Ascencio $(2004,198)$, elle cherche encore une fois à s'éloigner du texte source et rend mal caduc par mal de San Vito, un régionalisme de l'espagnol du Vénézuéla (v. Núñez / Pérez 1994 s.v. mal).

BILAN BIBL. : ø Pompilus 1961 ; TLF s.v. caduc B ; Fattier 1998, t. 2, p. 158 et carte 374 ; DRAE 2001 s.v. caduco ; « epilepsy» Valdman 2007 s.v. malkadi.

maringouin n. m. "petit insecte dont la piqûre, bien que bénigne, est redoutée".

« Il n'y a que la mare Zombi, mais c'est un marigot à maringouins : une eau pourrie comme une couleuvre morte, enroulée, une eau épaisse et sans force pour courir. » (p. 293)

Note de bas de page (de Jacques Roumain) : « Moustiques ».

« No hay más que el pantano Zombi, pero es un estanque para maringüinos ; un agua podrida como culebra muerta, enrollada, un agua espesa y sin fuerza para correr. » (p. 50)

Note de bas de page : « Mosquitos. »

- Type lexical hérité du tupi-guarani, traité dans Thibault 2009 (mot commun aux français d'Amérique et au français / créole antillais). Très bien représenté sous différentes formes en portugais (et en língua geral) ainsi qu'en français depuis l'époque coloniale, il est tout à fait inconnu en espagnol, d'où le caractère surprenant (car tout à fait artificiel) de la traduction proposée : maringüinos. Cela préserve toutefois le côté « saveur locale » de l'original, qui a été glosé lui aussi pour les lecteurs exogènes. 
Ascencio (2004, 149), se contente de traduire par l'équivalent diatopiquement neutre mosquitos.

BILAN BIBL. : Friederici 1947 s.v. maringouins; Thibault 2009, 100-102 (avec bibliographie); «mosquito» Valdman 2007 s.v. marengwen (mayengwen).

nègre a moué loc. subst. $\mathrm{m}$. en fonction apostrophe "mon homme, mon mari”.

« Puis, avec une grande tristesse et ses yeux sont pleins de larmes, elle dit doucement :

- O Bienaimé, nègre a moué...» (p. 268)

«Después, con una gran tristeza, y sus ojos que están llenos de lágrimas, dice dulcemente :

- ¡Oh! Bienaimé, negro josico parao...»

Note de bas de page : «De mal humor. (Hocico parado.) » ${ }^{9}$ (p. 3)

- Le syntagme nègre a moué appartient au créole et non au français régional ; cette locution de discours signifie tout simplement « mon nègre », donc en fait «mon homme, mon mari » dans le contexte antillais où le mot nègre n'est pas péjoratif et désigne tout homme de race noire. Il est très difficile de rendre la connotation d'une séquence en créole dans une traduction, mais le traducteur y arrive parfois assez bien dans la suite du livre en reproduisant l'accent caractéristique des noirs des Antilles lorsqu'ils parlent espagnol. C'est ce qu'il a tenté de faire ici, mais en changeant tout à fait le sens de l'original. S'il est bien vrai que josico parao représente une prononciation non standard d'une expression qui ne l'est pas davantage, le problème c'est que cette locution ne signifie pas du tout «à moi », mais bien «de mauvaise humeur» (comme nous le dit la note de bas de page). Cette bévue s'explique fort probablement par le fait que le traducteur aura lu mone (comme dans faire la mone) plutôt que moné, ce qui explique la traduction (ratée) josico parao.

Ascencio $(2004,122)$ traduit tout simplement (et correctement) par mi negro.

BILAN BIBL.: Sur moué dans les Antilles ainsi que dans les français d'Amérique, v. Thibault 2009, 80. - Sur nègre, cf. Thibault 2008, 285287.

\footnotetext{
${ }^{9}$ On trouve effectivement hocico (estirar el -) « demostrar enfado o malhumor mediante un gesto de la boca » dans Cárdenas Molina / Tristá Pérez / Werner (coord.) 2000.
} 
placé part. passé-adj. "en concubinage”.

« De mon temps, cette question des filles : c'était un tracas et une difficulté. Il fallait des manœuvres, des feintes, des parler-français, enfin toutes les macaqueries, toutes les simagrées et au bout du compte, tu te trouvais placé pour de bon et pour ainsi dire amarré comme un crabe, avec une case à bâtir, des meubles à acheter, sans compter la vaisselle. » (p. 288).

Note de bas de page (de Jacques Roumain) : «Plaçage : mariage paysan. »

«En mi tiempo esa cuestión de las muchachas era un embrollo y una dificultad. Se requerían maniobras, fingimientos, parlamentos, en fin, todas las macaquerías, todos los remilgos, $\mathrm{y}$, al fin de cuentas, te encontrabas colocado para siempre y, por así decirlo, amarrado como un cancrejo con una choza que construir, muebles que comprar, sin contar la vajilla. » (p. 42)

Note de bas de page : «Placage $[$ sic $]$ : matrimonio campesino. »

- On voit ici que le traducteur traduit non seulement le texte, mais aussi la note de bas de page (avec une faute d'orthographe). Le problème, c'est que si dans le texte original il y avait bien un rapport formel entre placé et plaçage, dans la traduction le lecteur hispanophone ne comprend pas du tout le rapport entre colocado et placage [sic], qui n'ont rien en commun du point de vue formel. Cela n'a aucun sens de traduire ainsi une note de bas de page de façon complètement aveugle. Quant à colocado, c'est une traduction littérale de placé, que l'on retrouve également dans Ascencio (2004, 145 et note 22$)$.

BILAN BIBL. : «Placer, v. pronom. ou passif. S'unir librement. Vivre en union libre [...]. » Pompilus 1961, 174-175; «1 common-law marriage, concubinage 2 cohabitation » Valdman 2007 s.v. plasay (plasaj).

Sor $n$. f. "(terme d'adresse précédant un prénom féminin et exprimant de l'affection ou du respect)".

« Je songe à Sor Mélie. La diablesse aurait pu mettre le feu à un bénitier. » (p. 288)

« Pienso en Sor Mélie, la diablesa hubiera prendido fuego a una pila de agua bendita. » (p. 42)

Note de bas de page : «Castellano en el original. » 
- Le traducteur s'est naïvement imaginé que ce mot était un emprunt à l'espagnol Sor, qui ne s'utilise que devant le prénom d'une religieuse. Or, dans le passage en question, il s'agit d'une femme très sexy et aguicheuse que le personnage finit par épouser! Le mot n'a rien à voir avec l'espagnol, c'est tout simplement le mot créole sor qui vient du mot français sœur et qui s'emploie en Haïti devant le prénom d'une femme qu'on aime bien. C'est un contresens que de le traduire par l'espagnol sor. Ascencio $(2004,145)$ a d'ailleurs opté pour une solution très simple (mais beaucoup plus juste), l'omission (« Me acuerdo de Mélie. »).

BILAN BIBL. : " my dear [term of affection or respect] Kouman sò Sofi ye? How is my friend Sophie doing ?» Valdman 2007 s.v. sè (sò).

tafia n. m. "liqueur provenant de la distillation des gros sucres, écumes et mélasses de la canne à sucre" (TLF).

« Pourtant on s'était à peine arrêté, le temps d'avaler une gorgée de tafia, de se détendre les reins - dans le corps c'est ce qu'il y a de plus récalcitrant, les reins. » (p. 273)

«Sin embargo apenas se habían detenido, sólo el tiempo de tragar un sorbo de tafia, de distender los riñones - en el cuerpo es lo que hay más recalcitrante. » (p. 12)

Note de bas de page : «Aguardiente de caña. »

- Antillanisme déjà ancien en français (première attestation : 1659, v. TLF) ; régionalisme de fréquence. En fait, ce type lexical figure également à la nomenclature du DRAE, de l'éd. de 1927 jusqu'à celle de 2001 (sans aucune mention de son origine française, cas de figure malheureusement très fréquent dans ce dictionnaire ; le DCECH de Corominas présente clairement le mot espagnol comme un emprunt au français). Comme le mot est très bien représenté dans la lexicographie espagnole, on peut considérer que la note de bas de page est superflue.

Ascencio (2004, 128 et note 8) reprend aussi la forme tafia comme telle dans sa version.

BILAN BIBL. : DRAE 1927 à 2001 ; DCECH ; TLF ; «locally produced raw rum » Valdman 2007 s.v. tafya. 


\section{Bilan}

L'échantillon que nous venons de présenter ne réunit qu'un tout petit pourcentage de tous les cas de diatopismes attestés dans Gouverneurs de la rosée (un dépouillement exhaustif nous a fourni en tout 928 exemples d'antillanismes dans cette source; nous n'en avons retenu que 29 dans le cadre de cette contribution, soit un peu plus de $3 \%$ ). Il ne s'agit donc pas d'un échantillon statistiquement représentatif des choix du traducteur, mais bien d'une sélection ayant pour but d'illustrer différents cas de figure.

D'abord, la méconnaissance du créole et du français haïtiens a provoqué un certain nombre d'erreurs assez grossières : ago n'est pas une onomatopée censée reproduire un éclat de rire, jouquer n'a rien à voir avec $y u c a$, Sor ne s'applique pas à une religieuse, un flamboyant n'est pas une espèce de petite tulipe, et enfin un nègre à moué ne fait pas la moue! On ne le dira jamais assez, la traduction d'œuvres littéraires francophones ne peut se faire qu'à l'aide d'excellents ouvrages de référence, à défaut desquels on n'hésitera pas à avoir recours à des personnes ressources, spécialistes du terrain.

Si l'on se reporte maintenant aux traductions qui, à tout le moins du point de vue référentiel, peuvent être considérées comme « correctes », on peut classer les choix du traducteur en deux grandes catégories : a) options " sourcières », c'est-à-dire essayant de coller au texte, à la langue et à la culture « source », en l'occurrence celles d'Haïti ; b) options " ciblistes », celles-là davantage tournées vers la langue et la culture " cibles ", c'est-àdire dans le cas qui nous occupe le monde hispanophone, lequel peut se scinder en de nombreux sous-ensembles diatopiques. Ce n'est pas notre propos de prendre position ici dans le débat opposant « sourciers » et « ciblistes » : ce sont pour nous des choix esthétiques et éthiques respectables mais dont il faut reconnaître qu'ils ont chacun leurs qualités et leurs défauts.

La traduction analysée abonde en choix « sourciers », que l'on peut regrouper en deux catégories : certains mots sont de purs artefacts, inventés ad hoc par le traducteur qui se contente d'hispaniser superficiellement un type lexical n'ayant jamais existé dans cette langue (ayupa, cadache, frangipanes, maringüino), voire de reprendre telle quelle la forme graphique du mot français (gros-bleu, loa). Quelques notes de bas de page aident toutefois le lecteur à s'y retrouver. D'autres mots n'existent que dans certaines variétés diatopiques ou diaphasiques de cette langue (a tiéa, bayahonda, caravanas, clerén, flamboyán, gallera, hierba de Guinea, jarina, latanero, macuto, tafia), éventuellement sous un autre graphie (cumbite correspond à l'espagnol convite). Plusieurs de ces mots sont propres aux variétés «caribeñas » de l'espagnol, ce qui est une excellente manière de réconcilier sourciers et ciblistes; il y avait là une chance à saisir, ce que le traducteur a su faire dans nombre de cas. L'emploi de calabacero pour traduire calebassier, alors que le mot espagnol désigne en fait la courge, est un autre exemple de procédé «sourcier» (on tient à tout prix à rester proche de la 
forme de départ, au mépris de la langue d'arrivée) mais qui affecte le sens seulement et non le lexème tout entier; de même, l'emploi métonymique de madrás pour désigner le foulard fait de ce tissu, inconnu de l'espagnol, préserve la forme de départ au prix d'un calque sémantique dans la langue d'arrivée. Le cas de candelabro pour « chandelier (esp. de cactus)» illustre la situation inverse : on s'éloigne de la forme de départ, mais c'est l'image qui est préservée. Dans certains cas, le mot traduisant est tiré de l'espagnol "général » mais correspond étymologiquement et formellement au mot de la langue de départ, ce qui permet d'obtenir une adaptation « sourcière » à bien peu de frais (casabe, don, mal caduco).

Les exemples « ciblistes », ou en tout cas les choix de traduction qui s'écartent résolument du mot de départ, sont très rares dans notre échantillon : nous n'avons relevé que colocado pour placé. De ce point de vue, la comparaison avec la traduction d'Ascencio 2004 laisse apparaître une situation diamétralement opposée : pour quelques choix « sourciers » (ago, casabe, clerén, cumbite, flamboyant, gros-bleue [sic], hierba de Guinea, loa, tafia), on trouve aussi dans cette version un grand nombre de solutions « ciblistes » (choza pour ayupa, abajo pour a tiéa, cujies pour bayahondas, tunas pour cadaches, taparo pour calabacero, trampas pour caravanas, lloviznita pequeña pour jarina, amapola pour frangipanes, posarse pour *yuquear, palmas pour latanero, mochila pour macuto, pañoleta pour madrás, mal de San Vito pour mal caduco, mosquitos pour maringüinos). La plupart de ces mots appartient à l'espagnol général, mais lorsqu'il s'agit de diatopismes ceux-ci sont propres à l'espagnol du Vénézuéla (cujies, taparo, mal de San Vito); on peut donc considérer que, si l'échantillon que nous avons analysé est représentatif, la « cible » de la traduction d'Ascencio est, très précisément, le lectorat vénézuélien. En revanche, la première traduction, celle de la Casa de las Américas, s'adresse à n'importe quel hispanophone (ses nombreux choix « sourciers » étant toujours glosés) mais tente de recréer tant bien que mal une ambiance linguistique antillaise grâce au recours à des lexies typiques de l'espagnol caribéen, démarche intéressante qui réconcilie deux idéologies à priori incompatibles (la source ou la cible) en allant puiser dans le stock lexical de la langue d'arrivée des diatopismes étymologiquement apparentés aux mots de la langue de départ.

André THIBAULT

Université de Paris Sorbonne (Paris IV)

UFR de Langue française

EA 4080 


\section{RÉFÉRENCES BIBLIOGRAPHIQUES}

ALEXIS, Jacques Stephen, 1955. Compère Général Soleil, Paris, Gallimard.

ARVEILLER, Raymond, 1963. Contribution à l'étude des termes de voyage en français (1505-1722), Paris, Editions d'Artrey.

ASCENCIO, Michaelle, 2004. Gobernadores del rocio y otros textos: Selección, traducción, prólogo, notas, cronología y bibliografía, Caracas, Biblioteca Ayacucho.

BARTHÈLEMI,Georges, 2007. Dictionnaire créole guyanais - français, Matoury, Guyane, Ibis Rouge Éditions.

BOLLÉE, Annegret, 2011. "Étymologies créoles. Contributions du Dictionnaire étymologique des créoles français d'Amérique (DECA) à l'histoire du vocabulaire régional antillais", dans A. THIBAULT (éd.), 2011, Le français dans les Antilles : études linguistiques, Paris, L'Harmattan, 31-50.

BOULAN, Henri René, 1934. Les mots d'origine étrangère en français (1650-1700), Paris / Amsterdam.

BRETON, R. P., 1999 [1665]. Dictionnaire caraïbe-français. Nouvelle édition sous la responsabilité de Marina Besada Paisa, Paris, Karthala.

CÁRDENAS MOLINA, G. / TRISTÁ PÉREZ, A. Ma / WERNER, R. (coord.), 2000. Diccionario del español de Cuba, Madrid, Gredos.

CHAUDENSON, Robert, 1974. Le lexique du parler créole de la Réunion, Paris, Champion.

CONFIANT, Raphaël, 2007. Dictionnaire créole martiniquais-français, Matoury, Guyane, Ibis Rouge Éditions.

CORDE = Corpus Diacrónico del Español (www.rae.es/...).

CORRALES, Cristóbal / CORBELLA, Dolores, 2001. Diccionario Histórico del Español de Canarias, La Laguna, Instituto de Estudios Canarios.

CORRALES, Cristóbal / CORBELLA, Dolores, 2009. Diccionario ejemplificado de canarismos, La Laguna, Instituto de Estudios Canarios.

DCECH = COROMINAS, Joan / PASCUAL, José A., 1980-1991. Diccionario crítico-etimológico castellano e hispánico, Madrid, Gredos.

DECOI = BOLLÉE, Annegret (dir.), 1993-2007. Dictionnaire étymologique des créoles français de l'Océan Indien, 4 vol., Hamburg, Helmut Buske.

DRAE $2001=$ Real Academia Española, 2001. Diccionario de la lengua española, Madrid, Espasa-Calpe ( $22^{\mathrm{e}}$ éd.).

DUF 1997 = Dictionnaire universel francophone, Paris, Hachette.

FAINE, Jules, 1974. Dictionnaire français-créole, Ottawa, Leméac.

FATTIER, Dominique, 1998. Contribution à l'étude de la genèse d'un créole: l'Atlas linguistique d'Haïti, cartes et commentaires, 6 vol., Villeneuve d'Ascq, ANRT (Agence Nationale de Reproduction des Thèses). 
FEW $=$ WARTBURG, Walther von, 1922-2002. Französisches Etymologisches Wörterbuch. Eine darstellung des galloromanischen sprachschatzes, Bonn / Leipzig / Bâle, Teubner / Klopp / Zbinden, 25 vol.

FRIEDERICI, Georg, 1947. Amerikanistisches Wörterbuch und Hilfwörterbuch für den Amerikanisten, Hamburg, Cram, De Gruyter.

GR 2008 = REY, Alain (dir.). Le Grand Robert de la langue française, version électronique. $2^{\mathrm{e}}$ éd. dirigée par Alain Rey du Dictionnaire alphabétique et analogique de la langue française de Paul Robert. Paris, Le Robert, 2008.

GRL $=$ Google Recherche de livres. Données bibliographiques et textuelles dont les sources sont les fonds numérisés de certaines bibliothèques et les ouvrages d'un programme de promotion de livres numérisés disponibles en ligne (http://books.google.fr/advanced_book_search?hl=fr).

GROSSCHMID, Pablo / ECHEGOYEN, Cristina, 1998. Diccionario de regionalismos de la lengua española, Barcelona, Juventud.

HÉNANE, René, 2004. Glossaire des termes rares dans l'ouvre d'Aimé Césaire, Paris, Jean Michel Place.

HOFFMANN, Léon-François (coord.), 2003. Jacques Roumain: Euvres complètes, édition critique, Madrid [...], ALLCA XX.

JOURDAIN, Élodie, 1956. Le vocabulaire du parler créole de la Martinique, Paris, Klincksieck.

LarBil 1998 = Grand Dictionnaire espagnol-français / français-espagnol, 1998. Paris, Larousse-Bordas.

LUDWIG, Ralph / MONTBRAND, Danièle / POULLET, Hector / TELCHID, Sylviane, 2002. Dictionnaire créole français (Guadeloupe). Nouvelle édition, Servedit / Jasor.

MORÍNIGO, Marcos A., 1993. Diccionario del español de América, Madrid, Anaya.

NPR 2008 = Le Nouveau Petit Robert. Dictionnaire alphabétique et analogique de la langue française. Nouvelle édition du Petit Robert de Paul Robert, texte remanié et amplifié sous la direction de Josette REYDEBOVE et Alain REY. Paris, Le Robert, 2007 [millésimé « 2008 »].

NÚÑEZ, Rocío / PÉREZ, Francisco J., 1994. Diccionario del habla actual de Venezuela: Venezolanismos, voces indigenas, nuevas acepciones, Caracas, Universidad Católica Andrés Bello / Centro de Investigaciones Lingüísticas y Literarias.

PAULEAU, Christine, 2007. Mots de Nouvelle-Calédonie, éléments de recherche sociolinguistique sur le français calédonien : inventaire lexicographique polylectal. Tome I. [Langue courante], t. II. [Flore et faune], Nouméa, SCEREN-CDP Nouvelle-Calédonie.

POMPILUS, Pradel, 1961. La langue française en Haïti, Paris, Institut des hautes études de l'Amérique latine.

RÉZEAU, Dominique / RÉZEAU, Pierre, 1995. De la Vendée aux Caraïbes : Le Journal (1878-1884) d'Armand Massé, missionnaire apostolique, Paris, L'Harmattan (2 vol.). 
RÉZEAU, Pierre, 2008. « Aspects du français et du créole des Antilles (notamment Saint-Domingue) à la fin du XVIII ${ }^{\mathrm{e}}$ siècle, d'après le témoignage d'un lexicographe anonyme », dans A. THIBAULT (coord.), Richesses du français et géographie linguistique: Recherches lexicographiques sur les variétés du français en France et hors de France, vol. 2, Bruxelles, De Boeck-Duculot (Champs linguistiques), 195-226.

RICHARD, Renaud (coord.), 2000. Diccionario de hispanoamericanismos no recogidos por la Real Academia, Madrid, Cátedra, 2000 ( $2^{\mathrm{a}}$ ed.)

ROUMAIN, Jacques, 1971. Gobernadores del rocio, La Habana, Casa de las Américas.

ROUMAIN, Jacques, Gouverneurs de la rosée (roman), dans Léon-François HOFFMANN (coord.), Jacques Roumain: Euvres complètes, édition critique, Madrid [...], ALLCA XX, 2003, pp. 255-396.

SCHWARZ-BART, Simone, 1979. Ti Jean L'Horizon, Paris, Seuil.

TELCHID, Sylviane, 1997. Dictionnaire du français régional des Antilles : Guadeloupe, Martinique, Paris, Bonneton.

THIBAULT, André, 2008. «Les régionalismes dans La Rue Cases-Nègres (1950) de Joseph Zobel» dans A. THIBAULT (coord.), Richesses $d u$ français et géographie linguistique: Recherches lexicographiques sur les variétés du français en France et hors de France, vol. 2, Bruxelles, De Boeck-Duculot (Champs linguistiques), 227-314.

THIBAULT, André, 2009. «Français d'Amérique et créole / français des Antilles : nouveaux témoignages », Revue de linguistique romane 73, 77-137.

$\mathrm{TLF}=$ Trésor de la langue française. Dictionnaire de la langue du XIX et $X X^{e}$ siècle (1789-1960), 1971-1994, édité par Paul Imbs (vol. 1-10), Paris, CNRS, et par Bernard Quemada (vol. 11-16), Paris, Gallimard.

TOURNEUX, Henry / BARBOTIN, Maurice, 1990. Dictionnaire pratique du créole de Guadeloupe (Marie-Galante), suivi d'un 'Index françaiscréole', 'nouvelle édition', Paris, Karthala.

VALDMAN, Albert et al. 2007. Haitian Creole-English Bilingual Dictionary, Indiana University, Creole Institute, Bloomington.

ZANOAGA, Teodor Florin, 2011. «Mots du vocabulaire de la magie et des superstitions dans un corpus de littérature antillaise contemporaine », dans M. J. KÜMMEL (éd.), Sprachvergleich und Sprachdidaktik: Beiträge zu den 19. GeSuS-Linguistiktagen Freiburg im Breisgau, 2.-4. März 2010, Hamburg, Dr. Kovač, 125-140 (Philologia, Sprachwissenschaftliche Forschungsergebnisse, Band 161). 